\title{
ORTHOGONAL ARRAYS OF STRENGTH 3 AND SMALL RUN SIZES
}

\author{
ANDRIES E. BROUWER, ARJEH M. COHEN, MAN V.M. NGUYEN
}

\begin{abstract}
All mixed (or asymmetric) orthogonal arrays of strength 3 with run size at most 64 are determined.
\end{abstract}

\section{INTRODUCTION}

In this paper we study mixed orthogonal arrays of strength 3 . Let $s_{1}, s_{2}, \ldots, s_{k}$ be a list of natural numbers, and for each $i$, let $Q_{s_{i}}$ be a set of size $s_{i}$. For natural numbers $t, N$, a multiset $\mathcal{F}$ of size $N$ whose elements are from $Q_{s_{1}} \times Q_{s_{2}} \times \cdots \times Q_{s_{k}}$ is called an orthogonal array of strength $t$, notation $O A\left(N, s_{1}, s_{2}, \cdots, s_{k}, t\right)$, if $t \leq k$, and, for every index set $I \subseteq\{1, \cdots, k\}$ of size at most $t$, each row of $\prod_{i \in I} Q_{s_{i}}$ occurs equally often in the projection of $\mathcal{F}$ onto the coordinates indexed by $I$.

We refer to the elements of $\mathcal{F}$ as runs, so $N$ is the number of runs of $\mathcal{F}$, also called its run size. The coordinates of $Q_{s_{1}} \times Q_{s_{2}} \times \cdots \times Q_{s_{k}}$ are called factors, so $k$ is the number of factors. Moreover, $s_{i}$ is called the level of the factor $i$. Instead of $s_{1}, s_{2}, s_{3}, \ldots$ we also write $2^{a} \cdot 3^{b} \cdot 4^{c} \cdots$, where the exponents $a, b, c, \ldots$ indicate the number of factors at level $2,3,4$, etc. An orthogonal array is called trivial if it contains each element of $Q_{s_{1}} \times Q_{s_{2}} \times \cdots \times Q_{s_{k}}$ the same number of times.

Orthogonal arrays of strength 2 have been studied extensively. In this paper, we study the case of strength $t=3$. We restrict ourselves mainly to $N \leq 64$.

Theorem 1. For every set of parameters $N, s_{1}, s_{2}, \ldots, s_{k}, t$ with $t=3$ and $N \leq 64$ such that an orthogonal array $O A\left(N, s_{1}, s_{2}, \cdots, s_{k}, t\right)$ exists, we construct at least one such array. More precisely, if $k=3$ such an array is trivial, and if $k>3 a$ construction is indicated in Table 1.

Of course the existence of $O A\left(N, s_{1}, s_{2}, \cdots, s_{k}, t\right)$ does not depend on the ordering of the parameters $s_{j}$, and we can take them in non-decreasing order if we wish.

Sometimes we shall find it useful to consider arrays with $s_{i}=1$ for some $i$. An $O A\left(N, 1, s_{1}, s_{2}, \cdots, s_{k}, t\right)$ is equivalent to an $O A\left(N, s_{1}, s_{2}, \cdots, s_{k}, t\right)$, provided $t \leq k$.

We prove the main theorem in three parts. In Section 2, we recall known facts, establish some upper bounds on the parameters of a strength 3 orthogonal array with run size at most 64 , and provide further basic material. In Section 3 we determine the feasible parameter sets. In Section 4, we provide explicit constructions for all cases mentioned in Table 1. In Section 5, we give the non-existence proof for parameters not appearing in the table.

Date: November 1, 2003.

Key words and phrases. Fractional factorial designs, orthogonal arrays. 


\begin{tabular}{|c|c|c|c|c|}
\hline$N$ & Levels & Existence & Construction & Nonexistence \\
\hline 8 & $2^{a}$ & $a \leq 4$ & $(\mathrm{H})$ & \\
\hline 16 & $2^{a} \cdot 4$ & $a \leq 3$ & (M) & \\
\hline 16 & $2^{a}$ & $a \leq 8$ & $(\mathrm{H})$ & \\
\hline 24 & $2^{a} \cdot 6$ & $a \leq 3$ & (M) & \\
\hline 24 & $2^{a} \cdot 3$ & $a \leq 4$ & (M) & $a=5$ \\
\hline 24 & $2^{a}$ & $a \leq 12$ & $(\mathrm{H})$ & \\
\hline 27 & $3^{b}$ & $b \leq 4$ & $(\mathrm{~L})$ & $b=5$ \\
\hline 32 & $2^{a} \cdot 8$ & $a \leq 3$ & (M) & \\
\hline 32 & $2^{a} \cdot 4^{2}$ & $a \leq 4$ & (AD) & \\
\hline 32 & $2^{a} \cdot 4$ & $a \leq 7$ & $(\mathrm{M})$ & \\
\hline 32 & $2^{a}$ & $a \leq 16$ & $(\mathrm{H})$ & \\
\hline 36 & $2^{2} \cdot 3^{2}$ & & $(\mathrm{~T})$ & \\
\hline 40 & $2^{a} \cdot 10$ & $a \leq 3$ & (M) & \\
\hline 40 & $2^{a} \cdot 5$ & $a \leq 6$ & $\left(\mathrm{X}_{1}\right)$ & $a=7$ \\
\hline 40 & $2^{a}$ & $a \leq 20$ & $(\mathrm{H})$ & \\
\hline 48 & $2^{a} \cdot 12$ & $a \leq 3$ & (M) & \\
\hline 48 & $2^{a} \cdot 4 \cdot 6$ & $a \leq 2$ & (M) & $a=3$ \\
\hline 48 & $2^{a} \cdot 6$ & $a \leq 7$ & (M) & \\
\hline 48 & $2^{a} \cdot 3 \cdot 4$ & $a \leq 4$ & $\left(\mathrm{X}_{2}\right)$ & $a=5$ \\
\hline 48 & $2^{a} \cdot 4$ & $a \leq 11$ & $(\mathrm{M})$ & \\
\hline 48 & $2^{a} \cdot 3$ & $a \leq 9$ & $\left(\mathrm{X}_{3}\right)$ & $a=10$ \\
\hline 48 & $2^{a}$ & $a \leq 24$ & $(\mathrm{H})$ & \\
\hline 54 & $3^{b} \cdot 6$ & $b \leq 3$ & (M) & $b=4$ \\
\hline 54 & $2^{a} \cdot 3^{b}$ & $a \leq 1, b \leq 5$ & $\left(\mathrm{X}_{4}\right)$ & $(a, b)=(0,6)$ \\
\hline 56 & $2^{a} \cdot 14$ & $a \leq 3$ & $(\mathrm{M})$ & \\
\hline 56 & $2^{a} \cdot 7$ & $a \leq 6$ & $(\mathrm{~J})$ & $a=7$ \\
\hline 56 & $2^{a}$ & $a \leq 28$ & $(\mathrm{H})$ & \\
\hline 60 & $2^{2} \cdot 3 \cdot 5$ & & $(\mathrm{~T})$ & \\
\hline 64 & $2^{a} \cdot 16$ & $a \leq 3$ & (M) & \\
\hline 64 & $2^{a} \cdot 4 \cdot 8$ & $a \leq 4$ & (M) & \\
\hline 64 & $2^{a} \cdot 8$ & $a \leq 7$ & (M) & \\
\hline 64 & $4^{c}$ & $c \leq 6$ & $(\mathrm{~L})$ & \\
\hline 64 & $2^{a} \cdot 4^{5}$ & $a \leq 2$ & $(\mathrm{~S})$ & $a=3$ \\
\hline 64 & $2^{a} \cdot 4^{4}$ & $a \leq 6$ & $\left(\mathrm{X}_{5}\right)$ & \\
\hline 64 & $2^{a} \cdot 4^{3}$ & $a \leq 8$ & $(\mathrm{~S})$ & $a=9$ \\
\hline 64 & $2^{a} \cdot 4^{2}$ & $a \leq 12$ & $(\mathrm{AD})$ & \\
\hline 64 & $2^{a} \cdot 4$ & $a \leq 15$ & $(\mathrm{M})$ & \\
\hline 64 & $2^{a}$ & $a \leq 32$ & $(\mathrm{H})$ & \\
\hline
\end{tabular}

TABLE 1. Parameters of orthogonal arrays of strength 3 with $N \leq 64$.

We briefly explain the table. In the second and third column, a set of levels $2^{a} 3^{b} 4^{c} \ldots$ is specified for which there exists an orthogonal array of strength 3 and run size $N$ as indicated in the first column. In the fourth column we give a reference to a construction. In the fifth column we give references to nonexistence proofs.

Some of the obtained arrays are given in the appendix. We use the terms "factor" and "column"; "fractional design" and "array" alternatively in the whole paper. 


\section{BASIC FACTS}

We start by fixing some notation and recalling well-known results which will be used throughout the paper.

\subsection{Taking the derived design.}

Proposition 2. Let $k \geq t \geq 1$. From an orthogonal array $O A\left(N, s_{1}, s_{2}, \cdots, s_{k}, t\right)$, we find, for each choice of column $j(1 \leq j \leq k)$ and each choice of symbol $s \in Q_{s_{j}}$, an orthogonal array $O A\left(N / s_{j}, s_{1}, \cdots, s_{j-1}, s_{j+1}, \cdots, s_{k}, t-1\right)$ by taking the runs that have an $s$ in the $j$-th position, and deleting that coordinate position.

Proof. This is immediately clear from the definition.

2.2. Rao's generalized bound. We recall the following well-known result (cf. [7], Theorem 9.4, [3], Theorem 2.6.2, [4]).

Theorem 3 (Rao's generalized bounds for mixed orthogonal arrays).

Let $k \geq t \geq 1$, and assume an orthogonal array $O A\left(N, s_{1}, s_{2}, \cdots, s_{k}, t\right)$ exists.

- If $t$ is even, then

$$
N \geq \sum_{j=0}^{t / 2} \sum_{|I|=j} \Pi_{i \in I}\left(s_{i}-1\right) .
$$

- Ift is odd, then a lower bound for $N$ is found by applying the above bound to the derived design $O A\left(N / s_{k}, s_{1}, s_{2}, \cdots, s_{k-1}, t-1\right)$, where $s_{k}$ is the largest among the $s_{j}$.

We will need this result for $t=2$ and $t=3$. Let us give the explicit form of the above bound in case $t=2$.

Corollary 4 (Rao's bound when $t=2$ ).

Assume an orthogonal array of the form $O A\left(N, s_{1}, s_{2}, \cdots, s_{k}, 2\right)$ exists. Then

$$
N \geq 1+\sum_{i=1}^{k}\left(s_{i}-1\right) .
$$

For instance, an $O A\left(N, 3^{5} .2,3\right)$ has derived design $O A\left(N / 3,3^{4} .2,2\right)$, so that $N / 3 \geq 1+4 .(3-1)+(2-1)=10$, that is, $N \geq 30$.

These bounds are very well known. It is possible to derive additional information in case equality holds (cf. [3] (5.4.6), [8]).

Proposition 5. Let $\mathcal{F}$ be an $O A\left(N, s_{1}, s_{2}, \cdots, s_{k}, 2\right)$ with $N=1+\sum\left(s_{i}-1\right)$. Then for any two runs we have $\sum_{j \in J} s_{j}=k-1$, where $J$ is the set of columns where the two runs agree.

Proof. Fix one run, say, the top row. Let the weight of row $r$ be $w=\sum_{j \in J(r)} s_{j}$, where $J(r)$ is the set of columns where row $r$ agrees with the top row. Let there be $n_{w}$ rows of weight $w$. Put $\sigma=\sum\left(s_{i}-1\right)$. Double counting (over all rows other than the top row) now yields

$$
\begin{gathered}
\sum_{w} n_{w}=N-1, \\
\sum_{w} w n_{w}=\sum_{i}\left(\frac{N}{s_{i}}-1\right) s_{i}=N k-\sigma-k,
\end{gathered}
$$


$\sum_{w} w^{2} n_{w}=\sum_{i \neq j}\left(\frac{N}{s_{i} s_{j}}-1\right) s_{i} s_{j}+\sum_{i}\left(\frac{N}{s_{i}}-1\right) s_{i}^{2}=N k(k-1)-(\sigma+k)^{2}+N(\sigma+k)$

so that

$$
\sum(w-c)^{2} n_{w}=\frac{N \sigma}{N-1}(N-1-\sigma)
$$

for $c=k-\sigma /(N-1)$. Since this last sum is nonnegative, $N \geq 1+\sigma$. In case equality holds, every weight is equal to $c$, and $c=k-1$.

For example, if an $O A\left(24,2^{21} .3,2\right)$ exists, then any two runs have 21 weighted agreements. Since all weights are even except that on the last column, any two runs agree in the last column. Contradiction, so no such array exists.

2.3. Divisibility condition for the run size. For any orthogonal array $\mathcal{F}$, the fact that derived designs exist implies a divisibility condition for the run size of $\mathcal{F}$.

Lemma 6. Let $k \geq t \geq 0$. In an $O A\left(N, s_{1}, s_{2}, \cdots, s_{k}, t\right)$, the run size $N$ is divisible by the least common multiple (lcm) of all numbers $\prod_{i \in I} s_{i}$ where $|I|=t$.

Proof. This says that the $t$ times derived design has an integral run size.

For example, in an $O A\left(N, 3^{5} \cdot 2^{1}, 3\right)$, the run size $N$ must be a multiple of $N_{0}=$ $\operatorname{lcm}(3 \cdot 3 \cdot 3,2 \cdot 3 \cdot 3)=54$.

2.4. Strength 3 transversal designs. Orthogonal arrays are equivalent to transversal designs. Here is the version appropriate for mixed arrays.

\section{Definition 7.}

$A$ transversal design (TD) of strength $t$, is a triple $(X, \mathcal{G}, \mathcal{B})$, where $X$ is a set of points; $\mathcal{G}$ is a partition of $X$ into groups; and $\mathcal{B}$ is a collection of subsets of $X$ called blocks such that:

(1) $t \leq|\mathcal{G}|$,

(2) every block meets every group in precisely one point,

(3) for every choice of $t$ distinct groups, all t-sets meeting each of those groups in precisely one point are contained in the same number of blocks.

If there are $e_{i}$ groups of size $s_{i}, i=1, \cdots, k$, the group type of $\mathcal{G}$ is defined to be the list $T:=\left[s_{1}^{e_{1}}, \cdots, s_{k}^{e_{k}}\right]$. Notice that a mixed orthogonal array $O A\left(N, s_{1}^{e_{1}} \cdots s_{k}^{e_{k}}, t\right)$ is equivalent to a transversal design of strength $t$ with group type $\left[s_{1}^{e_{1}}, \cdots, s_{k}^{e_{k}}\right]$ and with $N$ blocks. The "factors" (columns) and the "runs" (rows) in orthogonal arrays are "groups" and "blocks" in transversal designs.

For instance, an array $O A\left(72,2^{5} .3^{2}, 3\right)$ can be viewed as a transversal design on a set $X$ with 16 points, $G$ consists of 7 groups, $B$ has 72 blocks; the frequency of $(2,3,3)$-level triples is 4 , of $(2,2,3)$-level triples is 6 , and of $(2,2,2)$-level triples is 9 .

\section{Enumeration of FeAsible parameters}

First, we have to find all reasonable parameters for putative mixed orthogonal arrays, then we have to construct the arrays that exist, and finally we have to show nonexistence in the cases where no array exists.

Let us say that a parameter set is reasonable if it satisfies the divisibility conditions and the Rao bound. Let us say that a parameter set $O A\left(N, s_{1}, s_{2}, \cdots, s_{k}, t\right)$ is trivial if $k=t$. (Then a design with these parameters is necessarily trivial. Parameter sets with $\prod s_{i} \mid N$ allow a trivial design, but there may also be nontrivial solutions.) We find the following list. 
Lemma 8. The nontrivial reasonable parameter sets for mixed orthogonal arrays of strength 3 and run size at most 64 are

(1) $O A\left(4 m, 2^{a}, 3\right), 4 \leq a \leq 2 m, m$ even, $2 \leq m \leq 16$,

(2) $O A\left(4 m, 2^{3} \cdot m, 3\right), m$ even, $2 \leq m \leq 16$,

(3) $O A\left(8 m, 2^{a} . m, 3\right), 3 \leq a \leq 7,3 \leq m \leq 8$,

(4) $O A\left(8 m, 2^{a} .4 . m, 3\right), 2 \leq a \leq 4, m$ even, $4 \leq m \leq 8$,

(5) $O A\left(9 m, 3^{b} . m, 3\right), 3 \leq b \leq 4, m=3,6$,

(6) $O A\left(36,2^{2} .3^{2}, 3\right)$,

(7) $O A\left(48,2^{a} .3,3\right), 3 \leq a \leq 15$,

(8) $O A\left(48,2^{a} \cdot 3.4,3\right), 2 \leq a \leq 9$,

(9) $O A\left(48,2^{a} .4,3\right), 3 \leq a \leq 11$,

(10) $O A\left(54,2^{a} \cdot 3^{b}, 3\right), a=0,1, b \geq 1, a+b \geq 4, a+2 b \leq 19$,

(11) $O A\left(60,2^{2} .3 .5,3\right)$,

(12) $O A\left(64,2^{a} .4^{c}, 3\right), a \geq 0, c \geq 1, a+c \geq 4, a+3 c \leq 18$,

We see that Table 1 is complete, in the sense that it lists, for each reasonable nontrivial parameter set, either a construction or a nonexistence proof.

The following section has the constructions, the section after that the nonexistence proofs.

\section{Constructions}

\subsection{General constructions.}

\subsubsection{Trivial designs $(\mathrm{T})$.}

If $\prod s_{i} \mid N$, then a trivial design (a multiple of the full design) exists. This settles arrays $O A\left(36,2^{2} .3^{2}, 3\right)$ and $O A\left(60,2^{2} .3 .5,3\right)$.

Note that in these cases also nontrivial designs may exist. For example, up to isomorphism there are precisely 3 nonisomorphic $O A\left(36,2^{2} .3^{2}, 3\right)$ and 6 nonisomorphic $O A\left(60,2^{2} .3 .5,3\right)$.

\subsubsection{Hadamard construction $(\mathrm{H})$.}

If $H$ is a Hadamard matrix of order $k, 4 \mid k$, then $\left(\begin{array}{c}H \\ -H\end{array}\right)$ is an orthogonal array $O A\left(2 k, 2^{k}, 3\right)$.

Conversely, every $O A\left(2 k, 2^{k}, 3\right)$ is (equivalent to one) found this way.

\subsubsection{Taking multiples $(\mathrm{M})$.}

Given a design $O A\left(N, s_{1}, s_{2}, \cdots, s_{k}, t\right)$, we make an $O A\left(s N, s s_{1}, s_{2}, \cdots, s_{k}, t\right)$ for any positive integer $s$ by taking $s$ copies of the design, each copy with its own choice of symbols in the first column, and identical otherwise. Arrays $O A\left(8 s, 2^{3} .2 s, 3\right)$ are found in this way from $O A\left(8,2^{4}, 3\right)$. Also, $O A\left(24,2^{4} .3,3\right)$ is found in this way from $O A\left(8,2^{4} .1,3\right)$, that is, $O A\left(8,2^{4}, 3\right)$.

\subsubsection{Juxtaposition (J).}

More generally, given an $O A\left(N^{\prime}, s^{\prime}, s_{2}, \cdots, s_{k}, t\right)$ and an $O A\left(N^{\prime \prime}, s^{\prime \prime}, s_{2}, \cdots, s_{k}, t\right)$ we can construct an $O A\left(N^{\prime}+N^{\prime \prime}, s^{\prime}+s^{\prime \prime}, s_{2}, \cdots, s_{k}, t\right)$ by putting one above the other, making sure that both use disjoint symbol sets in the first column, and identical symbol sets in the other columns. In this way one obtains $O A\left(56,2^{a} .7,3\right)$ from $O A\left(40,2^{a} .5,3\right)$ and $O A\left(16,2^{a+1}, 3\right)$ for $a \leq 6$. 


\subsubsection{Linear codes (L).}

A $[n, k, d]_{q}$ code is a linear code of word length $n$, dimension $k$, and minimum distance $d$. The code words of the dual code (that has dimension $n-k$ ) form an $O A\left(N, q^{n}, d-1\right)$ with $N=q^{n-k}$. In particular, the $[4,1,4]_{3}$ repetition code gives an $O A\left(27,3^{4}, 3\right)$, and the $[6,3,4]_{4}$ hexacode gives an $O A\left(64,4^{6}, 3\right)$.

\subsubsection{Split (S).}

Given an $O A\left(N, s t, s_{1}, s_{2}, \cdots, s_{k}, t\right)$, we can make an $O A\left(N, s, t, s_{1}, s_{2}, \cdots, s_{k}, t\right)$ by replacing the symbols in $Q_{s t}$ by those of $Q_{s} \times Q_{t}$. In particular, a 4-level column can be split into two 2-level columns, and a 6 -level column can be split into a 2-level and a 3-level column.

\subsubsection{Agrawal-Dey construction (AD).}

Agrawal \& Dey [1] construct an $O A\left(8 m, 4^{2} .2^{2 m-4}, 3\right)$ given a Hadamard matrix of order $m \geq 4$. This yields $O A\left(32,2^{4} .4^{2}, 3\right)$ and $O A\left(64,2^{12} \cdot 4^{2}, 3\right)$. See also Gupta \& Nigam [5].

\subsection{Explicit constructions.}

4.2.1. $O A\left(40,2^{6} .5,3\right)\left(\mathrm{X}_{1}\right)$.

Let $M$ be the binary matrix

$\begin{array}{llllll}0 & 0 & 0 & 0 & 0 & 1 \\ 1 & 1 & 1 & 0 & 0 & 1 \\ 1 & 0 & 0 & 0 & 1 & 0 \\ 0 & 1 & 1 & 0 & 1 & 0 \\ 0 & 0 & 1 & 1 & 0 & 0 \\ 1 & 1 & 0 & 1 & 0 & 0 \\ 1 & 0 & 1 & 1 & 1 & 1 \\ 0 & 1 & 0 & 1 & 1 & 1\end{array}$

and let $M_{i}(0 \leq i \leq 4)$ be the matrix obtained from $M$ by fixing the first column and cyclically permuting the remaining five $i$ times. Let $N_{i}$ be the matrix obtained from $M_{i}$ by adding a constant column of all $i$ 's. Construct an $O A\left(40,2^{6} .5,3\right)$ by juxtaposing the five matrices $N_{i}$.

4.2.2. $O A\left(48,2^{4} .3 .4,3\right)\left(\mathrm{X}_{2}\right)$.

See the explicit matrix given in the appendix. There are precisely 19 such designs.

4.2.3. $O A\left(48,2^{9} .3,3\right)\left(\mathrm{X}_{3}\right)$.

See the explicit matrix given in the appendix. There are precisely 3 such designs.

4.2.4. $O A\left(54,2.3^{5}, 3\right)\left(\mathrm{X}_{4}\right)$.

See the explicit matrix given in the appendix. There are precisely 4 such designs.

4.2.5. $O A\left(64,2^{6} .4^{4}, 3\right)\left(\mathrm{X}_{5}\right)$.

Let $A$ and $B$ be the subsets $\{0,1\}$ and $\{2,3\}$ of the cyclic group $\mathbb{Z}_{4}$ of residues $\bmod$ 4. Take $Q_{4}=\mathbb{Z}_{4}$ and $Q_{2}=\{A, B\}$. One obtains an $O A\left(64,2^{6} .4^{4}, 3\right)$ by taking in $Q_{2}^{6} \times Q_{4}^{3}$ the vectors $\left(S_{1}, S_{2}, S_{3}, S_{4}, S_{5}, S_{6}, x_{1}, x_{2}, x_{3}, x_{4}\right)$ such that $x_{1}+$ $x_{2}+x_{3}+x_{4}=0$, and $\sum_{j=1}^{4} a_{j}^{(i)} x_{j} \in S_{i}(1 \leq i \leq 6)$, where the $a^{(i)}$ are the six vectors $(0,1,2,3),(0,1,3,2),(0,2,1,3),(0,2,3,1),(0,3,1,2),(0,3,2,1)$.

This is the unique design with these parameters. 


\section{NONEXISTENCE}

5.1. Nonexistence of $O A\left(24,2^{5} .3,3\right)$. Suppose we have an $O A\left(24,2^{5} .3,3\right)$. Consider the three derived designs at the ternary column: each is an $O A\left(8,2^{5}, 2\right)$.

Let there be $n_{i}$ rows in one of these $O A\left(8,2^{5}, 2\right)$ that have $i$ coincidences (agreements) with an arbitrary fixed given binary reference vector. Then

$$
\begin{gathered}
\sum n_{i}=8, \\
\sum i n_{i}=20, \\
\sum\left(\begin{array}{l}
i \\
2
\end{array}\right) n_{i}=20 .
\end{gathered}
$$

These three equations have only three possible solutions $\left(n_{i}\right)_{i=0}^{5}$ in nonnegative integers:

$$
\begin{aligned}
& A=[0,1,4,2,0,1] ; \\
& B=[1,0,2,4,1,0] ; \\
& C=[0,2,2,2,2,0] .
\end{aligned}
$$

For each of the three derived designs we find one of these three vectors, say $n^{(0)}$, $n^{(1)}$ and $n^{(2)}$. For the original design with 24 blocks we have an agreement count vector $t=n^{(0)}+n^{(1)}+n^{(2)}$ that also satisfies

$$
\sum\left(\begin{array}{l}
i \\
3
\end{array}\right) t_{i}=30
$$

Now $\sum\left(\begin{array}{l}i \\ 3\end{array}\right) n_{i}$ equals $12,8,10$ for $n=A, B, C$, respectively. We see that for each choice of reference vector there are two possibilities: $\left\{n^{(0)}, n^{(1)}, n^{(2)}\right\}=\{A, B, C\}$ or $n^{(0)}=n^{(1)}=n^{(2)}=C$.

Each time we pick a row of one of the three parts as reference vector we find a vector $A$ for that part, and hence a vector $B$ for another part, so that this other part contains the complementary row. Thus, the design is self-complementary, but none of the three parts contains a pair of complementary vectors.

Pick the reference vector in one of the parts, so that that part is of type A. Of the two vectors in the part of type $\mathrm{C}$ that agree in four positions with the reference vector, at least one has its complement in that same part, a contradiction.

5.2. Nonexistence of $O A\left(27,3^{5}, 3\right)$. First proof: an $O A\left(27,3^{5}, 3\right)$ would be a ternary code (not necessarily linear) of size 27 , word length 5 , and dual distance at least 4, contradicting the Delsarte linear programming bound.

Second proof: Nonexistence follows from Bush's inequality [2]. (Suppose one of the runs is 00000 . There are 6 runs with precisely two 0 's in the first two positions. These runs do not have 0's in the final two positions, and have mutually distinct tails. But only 4 possible tails are available.) 
5.3. Nonexistence of $O A\left(40,2^{7} .5,3\right)$ and $O A\left(56,2^{7} .7,3\right)$. We show nonexistence of $O A\left(8 m, 2^{7} . m, 3\right)$ for odd $m$.

There is a unique $O A\left(8,2^{7}, 2\right)$, constructed, e.g., by taking the seven characteristic vectors of the lines of the Fano plane and the all- 1 vector. This design has strength 2 , and if we consider three columns, then half of the combinations occur twice and half of the combinations do not occur at all, so that each 3-symbol pattern occurs an even number of times. Now consider an $O A\left(8 m, 2^{7} \cdot m, 3\right)$. All $m$ derived designs are $O A\left(8,2^{7}, 2\right)$, but every 3 -symbol pattern must occur $m$ times. This is impossible if $m$ is odd.

5.4. Nonexistence of $O A\left(48,2^{3} .4 .6,3\right)$. The derived design of an $O A\left(48,2^{3} .4 .6,3\right)$ at the 4 -level column is an $O A\left(12,2^{3} \cdot 6,2\right)$. But no such design exists. More generally we show nonexistence of $O A\left(4 m, 2^{3} .2 m, 2\right)$ for odd $m$. Such an array must have $2 m$ pairs of rows of which the binary part is complementary. Look at the $2 m$ rows with 0 in the first column. Of these, $m$ have a 1 in the second column, and $m$ have a 1 in the third column, so that second and third column agree in an even number of places in these $2 m$ rows. But if two bits agree, then their complements agree, so the total number of agreements between second and third column is divisible by 4 . But it is $2 m$. Contradiction.

5.5. Nonexistence of $O A\left(48,2^{5} .3 .4,3\right)$. The derived design of an $O A\left(48,2^{5} .3 .4,3\right)$ at the 4-level column is an $O A\left(12,2^{5} .3,2\right)$. But no such design exists.

Indeed, suppose we have such an array. We build a new array of dimensions 3 by 5 and symbols in $\{A, B, C\}$. For each of the three derived $O A\left(4,2^{5}, 1\right)$ of our $O A\left(12,2^{5} .3,2\right)$ we make a row of the new array, by writing $A$ for each column $(0011)^{T}$ or $(1100)^{T}, B$ for each column $(0101)^{T}$ or $(1010)^{T}$, and $C$ for each column $(0110)^{T}$ or $(1001)^{T}$.

In $O A\left(4,2^{5}, 1\right)$, if two columns get different symbols, then every possible pair occurs once. If two columns get the same symbol then every possible pair occurs an even number of times.

Now the fact that every possible binary pair in $O A\left(12,2^{5} .3,2\right)$ occurs 3 times means that every two columns in the new array differ in 1 or 3 places.

But there is no 3-by-5 ternary array in which every two columns have an odd

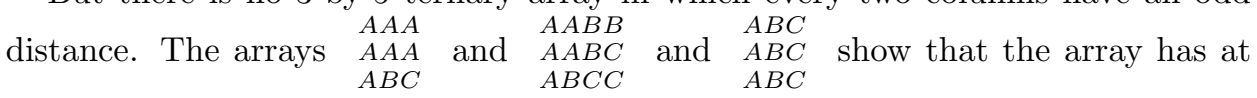
most three columns when there are three columns pairwise at distance 1 , or three columns pairwise at distance 3 , and at most four columns otherwise.

5.6. Nonexistence of $O A\left(48,2^{10} .3,3\right)$.

This follows by exhaustive computer search.

5.7. Nonexistence of $O A\left(54,3^{4} .6,3\right)$.

This follows by exhaustive computer search, either directly, or by observing that by construction (S) one gets $O A\left(54,3^{5} .2,3\right)$, and none of the four nonisomorphic such arrays allows going back.

5.8. Nonexistence of $O A\left(54,3^{6}, 3\right)$.

This follows by the Delsarte linear programming bound. See also [6]. 
5.9. Nonexistence of $O A\left(64,2^{3} \cdot 4^{5}, 3\right)$.

Suppose an $O A\left(64,2^{3} .4^{5}, 3\right)$ exists. The derived designs at a 4 -level factor are designs $O A\left(16,2^{3} .4^{4}, 2\right)$, but there is a unique such design.

The unique $O A\left(16,2^{3} .4^{4}, 2\right)$ can be obtained from the unique $O A\left(16,4^{5}, 2\right)$ by letting the three groups of size 2 be the three partitions into two pairs of the group of size 4 that they replace.

$O A\left(16,4^{5}, 2\right)$ can be viewed as the projective plane of order 4 minus a point $P$, where the groups are the five lines on $P$, and the blocks are the 16 lines not on $P$. Consequently, $O A\left(16,2^{3} .4^{4}, 2\right)$ can be viewed as the affine plane of order 4 , with the groups being the four lines in one direction $d$ and the three partitions of the set of directions different from $d$ into two disjoint pairs.

Note that parallel lines have the same direction, and hence are incident with the same pairs. Now take a run 00000000 of $O A\left(64,2^{3} \cdot 4^{5}, 3\right)$. For each of the five choices of 0 in one of the 4-level columns there are three lines parallel to 0000000 in the derived design, and each of these starts with 000. Altogether the binary triple 000 is covered 16 times, but it should be covered only 8 times.

5.10. Nonexistence of $O A\left(64,2^{9} .4^{3}, 3\right)$.

Consider a design $O A\left(16,2^{9} \cdot 4^{2}, 2\right)$. It has equality in the Rao bound, and Proposition 5 tells us that any two rows agree in precisely 5 places, when agreements in 4-level columns are counted twice.

Now consider $O A\left(64,2^{9} .4^{3}, 3\right)$, and without loss of generality assume that its top row is 00000000000 . Then that row has pattern $0^{9} 0^{2}, 9$ rows have pattern $\left(0^{3} 1^{6}\right)\left(0^{2} *^{1}\right), 27$ rows have pattern $\left(0^{5} 1^{4}\right)\left(0^{1} *^{2}\right)$, and 27 rows have pattern $\left(?^{9}\right)\left(*^{3}\right)$, where $*$ denotes any nonzero symbol.

Let there be $m_{i}$ rows with $i$ zeros in binary columns. Two rows that agree in a quaternary column have binary parts at even Hamming distance. Since the graph on the 64 rows defined by "agreeing in some quaternary column" is connected, it follows that any two binary parts have even Hamming distance, so that $m_{i}$ is only nonzero for odd $i$. Also $m_{9} \geq 1$. We obtain

$$
\begin{gathered}
\sum m_{i}=64, \\
\sum i m_{i}=9.32=288, \\
\sum\left(\begin{array}{l}
i \\
2
\end{array}\right) m_{i}=\left(\begin{array}{l}
9 \\
2
\end{array}\right) \cdot 16=576, \\
\sum\left(\begin{array}{l}
i \\
3
\end{array}\right) m_{i}=\left(\begin{array}{l}
9 \\
3
\end{array}\right) \cdot 8=672 .
\end{gathered}
$$

This has solution $\left(m_{1}, m_{3}, m_{5}, m_{7}, m_{9}\right)=(3,18,36,6,1)$ or $(4,14,42,2,2)$.

If $m_{9}=2$ then we have rows say $0^{9} 000$ and $0^{9} 111$. Now the row $?^{9} 110$ must have both 3 and 5 zeros in binary columns. Contradiction.

It follows that $\left(m_{1}, m_{3}, m_{5}, m_{7}, m_{9}\right)=(3,18,36,6,1)$. Subtract the known part, for the rows that have a 0 in the quaternary part. They contribute $(0,9,27,0,1)$, so that $(3,9,9,6,0)$ is contributed by rows without 0 in the quaternary part.

Look at the three rows with precisely one 0 . They mutually agree in at least seven binary positions, hence have no agreements in quaternary positions, and w.l.o.g. we have the rows 011111111111 and 101111111222 and 110111111333 . Any row with seven zeros in binary positions has at most three agreements with each of these 
three rows, so in its quaternary part every symbol occurs zero or two times. But 3 is odd, contradiction.

\section{Closing Remark}

The next interesting value for the run size is $N=72$. We have partial results only.

\section{REFERENCES}

[1] Agrawal, V., Dey, A., 1983. Orthogonal resolution-IV designs for some asymmetrical factorials. Technometrics 25, 197-199.

[2] Bose, R.C., Bush, K.A., 1952. Orthogonal arrays of strength 2 and 3. Ann. Math. Statistics 23, 508-524.

[3] Dey, A., Mukerjee, R., 1999. Fractional Factorial Plans. John Wiley \& Sons, New York.

[4] Diestelkamp, W.S., 1998. Parameter inequalities for orthogonal arrays with mixed levels. Department of Mathematics, University of Dayton. To appear in Designs, Codes \& Cryptography 33 (2004) 187-197.

[5] Gupta, V.K., Nigam, A.K., 1985. A class of asymmetrical orthogonal resolution IV designs. J. Statist. Plann. Inf. 11, 381-383.

[6] Hedayat, A.S., Seiden, E., Stufken, J., 1997. On the maximal number of factors and the enumeration of 3-symbol orthogonal arrays of strength 3. Journal of Statistical Planning and Inference 58, 43-63.

[7] Hedayat, A.S., Sloane, N.J.A., Stufken, J., 1999. Orthogonal Arrays, Springer, New York.

[8] Mukerjee, R., Wu, C.F.J., 1995. On the existence of saturated and nearly saturated asymmetrical orthogonal arrays. Ann. Statist. 23, 2102-2115.

[9] Sloane, N.J.A., Web site on Hadamard matrices. http://www.research.att.com/ njas/hadamard/.

[10] Wu, C.F.J., 1992. Nearly orthogonal arrays with mixed levels and small runs. Technometrics $34,409-422$. 
ApPEndix: List of SOME STRENGTH 3 ARRAYS

For each explicit construction, we give an array. To save space, we give the transposed matrix: runs are columns here.

Construction $\left(\mathbf{X}_{1}\right)$. $O A\left(40,5 \cdot 2^{6}, 3\right)$.

0000000011111111222222223333333344444444

0110011001100110011001100110011001100110

0101010101011010000011110011001111000011

0101101000001111001100111100001101010101

0000111100110011110000110101010101011010

0011001111000011010101010101101000001111

1100001101010101010110100000111100110011

Construction $\left(\mathbf{X}_{2}\right) . O A\left(48,4 \cdot 3 \cdot 2^{4}, 3\right)$.

00000000000111111111111222222222222333333333333

000011112222000011112222000011112222000011112222

001100110011001100110011001100110011001100110011 010101010101010101010101010101010101010101010101 001111001001110000110110001111001001110000110110 010110011010101001100101101001100101010110011010

Construction $\left(\mathbf{X}_{3}\right) . O A\left(48,3.2^{9}, 3\right)$.

00000000000000001111111111111112222222222222222 000000001111111100000000111111110000000011111111 000011110000111100001111000011110000111100001111 000011111111000001110001000101110111000100010111 001100110011001100010111011100011011001000101011 001100111100110010101010001100110101010101101001 010101010101010100101011110101001110100001001101 010101011010101011001100010101010011001111001100 011010010110100101001101101100101101010010001110 011010011001011010010110100101100110100110101010

Construction $\left(\mathbf{X}_{4}\right)$. $O A\left(54,3^{5} \cdot 2,3\right)$.

000000000000000000111111111111111111222222222222222222

000000111111222222000000111111222222000000111111222222

001122001122001122001122001122001122001122001122001122

001122120201120201120201001122120201120201120201001122

010212121020202101121020021201010212202101010212120102

011001011001100110100110100101011001011001100110010110 
Construction $\left(\mathbf{X}_{5}\right) . O A\left(64,4^{4} \cdot 2^{6}, 3\right)$.

0000000000000000111111111111111122222222222222223333333333333333

0000111122223333000011112222333300001111222233330000111122223333

0123012301230123012301230123012301230123012301230123012301230123

0123103223103201103201233201231023103201103201233201231001231032

0011110000111100110000111100001111000011001111000011110011000011

0011110011000011110000110011110000111100001111001100001111000011 0101010110101010101010100101010110011001011001100110011010011001 0101101010010110010110101001011010100101011010011010010101101001 0110011010011001100110010110011001010101101010101010101001010101 0110100101011010011010010101101010010110101001011001011010100101 